University of New Orleans

ScholarWorks@UNO

$11-1986$

\title{
Thin-film beam splitter that reflects light as a half-wave retarder and transmits it without change of polarization: application to a Michelson interferometer
}

R. M.A. Azzam

University of New Orleans, razzam@uno.edu

Follow this and additional works at: https://scholarworks.uno.edu/ee_facpubs

Part of the Electrical and Electronics Commons, and the Optics Commons

\section{Recommended Citation}

R. M. A. Azzam, "Thin-film beam splitter that reflects light as a half-wave retarder and transmits it without change of polarization: application to a Michelson interferometer," J. Opt. Soc. Am. A 3, 1803-1808 (1986)

This Article is brought to you for free and open access by the Department of Electrical Engineering at ScholarWorks@UNO. It has been accepted for inclusion in Electrical Engineering Faculty Publications by an authorized administrator of ScholarWorks@UNO. For more information, please contact scholarworks@uno.edu. 


\title{
Thin-film beam splitter that reflects light as a half-wave retarder and transmits it without change of polarization: application to a Michelson interferometer
}

\author{
R. M. A. Azzam \\ Department of Electrical Engineering, University of New Orleans, Lakefront, New Orleans, Louisiana 70148
}

Received March 3, 1986; accepted May 7, 1986

\begin{abstract}
The refractive index $n_{1}$ of a transparent layer of quarter-wave optical thickness coating a transparent substrate of refractive index $n_{2}$ can be chosen to produce half-wave retardation (HWR) in reflection and no change of polarization in refraction at any angle of incidence $\phi$. The function $n_{1}\left(\phi, n_{2}\right)$, and the associated polarization-independent reflectance of the film-substrate system $\mathcal{R}\left(\phi, n_{2}\right)$ are determined. Such a coated surface can be used as a beam splitter with excellent characteristics (e.g., split fractions that do not depend on source polarization, a split beam whose polarization is identical to that of the incident beam and operation over a wide range of incidence angles). A concrete example of a coated Ge-slab beam splitter for $10.6-\mu \mathrm{m}$ radiation at $\phi=45^{\circ}$ is given. The beam-splitter face of the slab is coated with the HWR layer, and the exit face is coated with a double layer that produces total refraction without change of polarization. Such a beam splitter is tolerant to film-thickness errors and is reasonably achromatic over a small (e.g., 10-11- $\mu \mathrm{m}$ ) wavelength range. When used in a Michelson interferometer this beam splitter renders its operation totally independent of source polarization.
\end{abstract}

\section{INTRODUCTION}

I have recently ${ }^{1}$ shown that the reflection and refraction of light of wavelength $\lambda$ at an angle of incidence $\phi$ by the interface between two dielectric (linear, homogeneous, isotropic, and nonmagnetic) media of refractive indices $N_{0}$ and $N_{2}$ can be made completely insensitive to polarization by introducing an intermediate layer of refractive index

$$
N_{1}=\left(N_{0} N_{2}\right)^{1 / 2}
$$

and of thickness

$$
d=(\lambda / 4)\left(N_{1}^{2}-N_{0}^{2} \sin ^{2} \phi\right)^{-1 / 2}
$$

or an odd integral multiple thereof. This has led to a simple polarization-independent beam splitter ${ }^{2}$ (PIBS) that divides the incident beam into two beams with constant reflectance and transmittance and with polarization identical to the incident polarization, for all incident polarizations. Equations (1) and (2) guarantee that

$$
R_{p}=R_{s}
$$

and

$$
T_{p}=T_{s},
$$

where $R_{\nu}$ and $T_{\nu}$ are the complex-amplitude reflection and transmission coefficients for the $\nu=p$ and $\nu=s$ polarizations, parallel and perpendicular to the plane of incidence, respectively.

In this paper I show that, for given $N_{0}, N_{2}$, and $\phi$, the refractive index of a layer of quarter-wave optical thickness, given by Eq. (2), can be chosen such that the following modified set of equations,

$$
R_{p}=-R_{s}
$$

and

$$
T_{p}=T_{s},
$$

is satisfied simultaneously, instead of Eqs. (3) and (4). Equations (5) and (6) indicate that the layer reflects light as a half-wave retarder (HWR) and transmits it with no change of polarization. This leads to a new beam splitter with several significant characteristics: (1) the split fractions of the input power carried by the reflected and transmitted beams are constant independent of the input polarization; (2) the polarization state of the transmitted beam is always identical to that of the incident beam; (3) the reflected polarization is simply related to the incident polarization by a transformation that is exactly that produced by an HWR with fast and slow axes aligned with the $p$ and $s$ directions ${ }^{3}$; (4) the reflectance of this beam splitter is considerably higher, at lower angles of incidence, than that of the beam splitter of Refs. 1 and 2; and (5) all the preceding four properties remain virtually unchanged as the angle of incidence is varied from zero up to (and a little beyond) the design angle $\phi$. Furthermore, a specific example of a coated Ge slab for 10.6$\mu \mathrm{m}$ radiation (see Section 5) reveals that (6) the design is tolerant to appreciable film-thickness errors and, by consequence, (7) the beam splitter is reasonably achromatic over a limited (e.g., 10-11- $\mu \mathrm{m}$ ) wavelength range.

Finally, we demonstrate in Section 6 that this new beam splitter, when used in a Michelson interferometer, renders it completely independent of source polarization.

\section{FILM REFRACTIVE INDEX}

For a layer of thickness given by Eq. (2), $R_{p}$ and $R_{s}$ become real and are given by ${ }^{1}$

$$
\begin{aligned}
R_{p} & =\left(N_{1}{ }^{4} S_{0} S_{2}-N_{0}{ }^{2} N_{2}{ }^{2} S_{1}{ }^{2}\right) /\left(N_{1}{ }^{4} S_{0} S_{2}+N_{0}{ }^{2} N_{2}{ }^{2} S_{1}{ }^{2}\right), \\
R_{s} & =\left(S_{0} S_{2}-S_{1}^{2}\right) /\left(S_{0} S_{2}+S_{1}{ }^{2}\right)
\end{aligned}
$$

where

$$
S_{m}=\left(N_{m}{ }^{2}-N_{0}^{2} \sin ^{2} \phi\right)^{1 / 2}, \quad m=0,1,2 .
$$




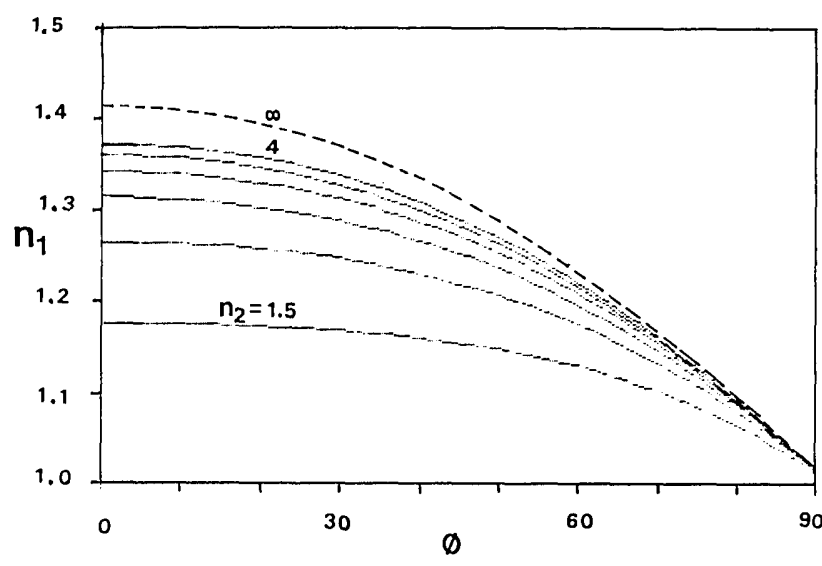

Fig. 1. Refractive index $n_{1}\left(\phi, n_{2}\right)$ [Eq. (12)] of a quarter-wave layer that, when deposited on a substrate of refractive index $n_{2}$, produces the half-wave retardation in reflection and no change of polarization in refraction at the angle of incidence $\phi . n_{1}$ is plotted versus $\phi$ with $n_{2}$ as a parameter taking values from 1.5 to 4 in equal steps of 0.5 . Both $n_{1}$ and $n_{2}$ are normalized with respect to the ambient (incidence-medium) refractive index [Eq. (11)]. The dashed curve at the top represents the limit of $n_{1}$ as $n_{2} \rightarrow \infty$ [Eq. (14)].

Substituting Eqs. (7) and (8) into Eq. (5) gives, after simplification,

$$
N_{0} N_{2} S_{1}^{2}=N_{1}^{2} S_{0} S_{2}
$$

If $S_{m}(m=0,1,2)$ in Eq. (10) is replaced by its value from Eq. (9), and the normalization

$$
n_{1}=N_{1} / N_{0}, \quad n_{2}=N_{2} / N_{0}
$$

is used, the resulting equation can be solved for $n_{1}^{2}$ to give

$$
n_{1}^{2}=n_{2} \sin ^{2} \phi /\left[n_{2}-\cos \phi\left(n_{2}^{2}-\sin ^{2} \phi\right)^{1 / 2}\right] .
$$

Equation (12) gives an explicit solution for the refractive index $n_{1}$ of a quarter-wave layer that reflects light as an HWR in terms of the substrate refractive index $n_{2}$ and the angle of incidence $\phi$. The function $n_{1}=f\left(\phi, n_{2}\right)$, Eq. (12), is represented graphically in Fig. 1 by plotting $n_{1}$ versus $\phi$ with $n_{2}$ as a constant parameter taking values from 1.5 to 4 in equal steps of 0.5 . At exact normal incidence, $\phi=0$, the right-hand side (rhs) of Eq. (12) takes the form $0 / 0$, and $n_{1}$ becomes indeterminate. This mathematical answer is justified physically because, when $\phi=0, R_{p}=-R_{s}$ in the Nebraska (Muller) conventions ${ }^{4}$ for any optically isotropic surface, coated or uncoated, and HWR is obtained independent of $n_{1}$. However, as $\phi \rightarrow 0$ the rhs tends to a specific limit given by

$$
n_{1}^{2}=2 n_{2}^{2} /\left(n_{2}^{2}+1\right) \text {. }
$$

For a given $n_{2}, n_{1}$ decreases monotonically from a maximum given by Eq. (13) to 1, as $\phi$ is increased from $0^{\circ}$ to $90^{\circ}$, respectively, as is evident from Fig. 1. At any given $\phi$ an upper bound, $\hat{n}_{1}$, on $n_{1}$ is obtained by taking the limit of the rhs of Eq. (12) as $n_{2} \rightarrow \infty$; this gives

$$
\hat{n}_{1}=\sqrt{2} \cos (\phi / 2) .
$$

Thus the normalized film refractive index $n_{1}$ is always $<\sqrt{2}$ $=1.414$.

For the most practical case when the medium of incidence is air $\left(N_{0}=1\right)$, values of $n_{1}=N_{1} \lesssim 1.2$ become unrealizable by a thin solid film. ${ }^{5}$ From Fig. 1 it follows that the sub- strate must be optically dense, $n_{2} \gtrsim 2$, and high angles of incidence should be avoided, $\phi<60^{\circ}$.

If the numerator and denominator of the rhs of Eq. (12) are divided by $n_{2}$ and Snell's law is applied, we obtain

$$
n_{1}=\sin \phi /\left(1-\cos \phi \cos \phi^{\prime \prime}\right)^{1 / 2},
$$

where $\phi^{\prime \prime}=\arcsin \left(\sin \phi / n_{2}\right)$ is the angle of refraction of light into the substrate. Equation (15) gives the required film refractive index in terms of the angles of incidence and refraction $\phi$ and $\phi^{\prime \prime}$. If we denote by $\phi^{\prime}$ the angle of refraction in the film, the following interesting result is obtained from Eq. (15):

$$
\cos \phi^{\prime}=\left(\cos \phi \cos \phi^{\prime \prime}\right)^{1 / 2} .
$$

Therefore, for the film that produces HWR in reflection, the cosine of the angle of refraction in the film equals the geometric mean of the cosines of the angles of incidence and refraction into the substrate. In Appendix A, Eq. (12) is solved for $\phi$ for given $n_{1}$ and $n_{2}$.

\section{REFLECTION COEFFICIENTS}

Let us write

$$
R_{s}=-R_{p}=R \text {. }
$$

If we substitute Eq. (10) into Eq. (8), we get

$$
R=\left(n_{2}-n_{1}^{2}\right) /\left(n_{2}+n_{1}^{2}\right),
$$

which is a simple expression for the (complex) amplitude reflection coefficient of the HWR film-substrate system. To write $R$ in terms of $n_{2}$ and $\phi, n_{1}^{2}$ in Eq. (18) is replaced by its value from Eq. (12); this gives

$$
R=\frac{\left(n_{2}-\sin ^{2} \phi\right)-\left(n_{2}{ }^{2}-\sin ^{2} \phi\right)^{1 / 2} \cos \phi}{\left(n_{2}+\sin ^{2} \phi\right)-\left(n_{2}{ }^{2}-\sin ^{2} \phi\right)^{1 / 2} \cos \phi} .
$$

The intensity (power) reflectance,

$$
\mathcal{R}=|R|^{2},
$$

is, of course, the same for the $p$ and $s$ (hence for all incident) polarizations. Figure 2 shows $\mathcal{R}\left(\phi, n_{2}\right)$ as a function of $\phi$ for constant values of $n_{2}$ from 1.5 to 4 in equal steps of 0.5 , i.e., corresponding to the data of Fig. 1.

The normal-incidence limit on $R$ is obtained from Eqs. (13) and (18):

$$
R(0)=\left[\left(n_{2}-1\right) /\left(n_{2}+1\right)\right]^{2},
$$

and the corresponding intensity reflectance is

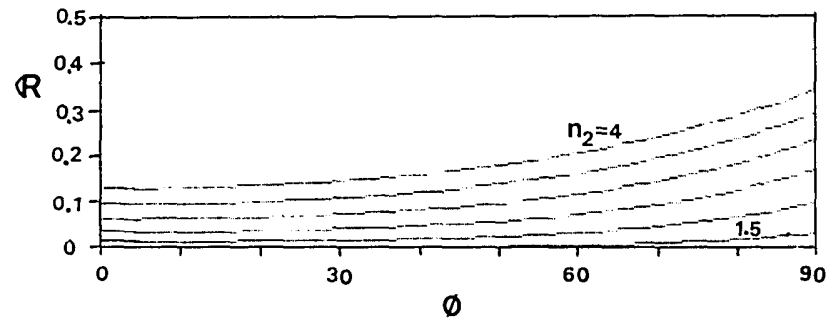

Fig. 2. Beam-splitter reflectance $\mathcal{R}\left(\phi, n_{2}\right)$ [Eqs. (19) and (20)] as a function of the angle of incidence $\phi$, with the substrate normalized refractive index $n_{2}$ as a parameter taking values from 1.5 to 4 in equal steps of 0.5 . The beam splitter uses a thin film whose properties appear in Fig. 1. 


$$
\begin{aligned}
\mathscr{R}(0) & =\left[\left(n_{2}-1\right) /\left(n_{2}+1\right)\right]^{4} \\
& =\overline{\mathcal{R}}^{2}(0),
\end{aligned}
$$

where $\bar{R}(0)$ is the (film-free) bare-substrate reflectance at normal incidence. Equation (23) is another curious result and indicates that the layer required to produce HWR near normal incidence reduces the substrate reflectance to its square.

The grazing-incidence limit is obtained by setting $n_{1}=1$ in Eq. (18):

$$
\begin{aligned}
R\left(90^{\circ}\right) & =\left(n_{2}-1\right) /\left(n_{2}+1\right), \\
\mathcal{R}\left(90^{\circ}\right) & =\left[\left(n_{2}-1\right) /\left(n_{2}+1\right)\right]^{2} \\
& =\overline{\mathcal{R}}(0) .
\end{aligned}
$$

Equations (25) and (26) indicate that the normal-incidence reflectance of the bare substrate represents an upper bound on the reflectance of the (all-dielectric) film-substrate HWR. In Fig. 2 the highest reflectance occurs for $n_{2}=4$ as $\phi$ $\rightarrow 90^{\circ}$ and is equal to $(3 / 5)^{2}$, or $36 \%$. Figure 2 also shows that, for a given $n_{2}$, the rise of $\mathcal{R}$ with $\phi$ is relatively moderate. Recall from Section 2 that for $\phi>60^{\circ}, n_{1}$ becomes too close to 1 to be realizable by a thin solid film, so that this range of high angles is not useful. ${ }^{6}$

The reflectance levels attainable at $\phi<60^{\circ}$ are adequate for beam splitters and are considerably higher than those possible with the PIBS of Refs. 1 and 2 in this range of incidence angles. For example, by using a Ge substrate, $n_{2}$ $=4$, the reflectances of the PIBS at $45^{\circ}$ and $60^{\circ}$ are 1.30 and $6.22 \%$, as compared with 17.41 and $21.51 \%$, respectively, for the present device. The reflectance gain at $45^{\circ}$ exceeds 1 order of magnitude. The beam splitter application will be considered in detail in Section 5.

\section{TRANSMISSION COEFFICIENTS}

For a layer of quarter-wave optical thickness, Eq. (2), the complex amplitude transmission coefficients for the $p$ and $s$ polarizations are given by ${ }^{7}$

$$
\begin{aligned}
& T_{p}=-j 2\left(N_{0} N_{1}{ }^{2} N_{2}\right) S_{0} S_{1} /\left(N_{1}{ }^{4} S_{0} S_{2}+N_{0}{ }^{2} N_{2}{ }^{2} S_{1}{ }^{2}\right), \\
& T_{s}=-j 2 S_{0} S_{1} /\left(S_{0} S_{2}+S_{1}{ }^{2}\right),
\end{aligned}
$$

where $S_{m}$ is defined by Eq. (9) and $j=\sqrt{-1}$. When the layer is chosen to make $R_{p}=-R_{s}$, i.e., the film-substrate system acts as an HWR in reflection, Eq. (10) is satisfied. Substitution of Eq. (10) into Eqs. (27) and (28) leads to

$$
T_{p}=T_{s}=T,
$$

where

$$
T=-j 2 n_{1}{ }^{2} \cos \phi /\left(n_{2}+n_{1}{ }^{2}\right)\left(n_{1}{ }^{2}-\sin ^{2} \phi\right)^{1 / 2} .
$$

The normalized film and substrate refractive indices $n_{1}$ and $n_{2}$, Eqs. (11), are interrelated by Eq. (12).

Equation (29) leads to the important conclusion that the wave is refracted into the substrate without change of polarization. This in turn, suggests that this coated dielectric surface be used as a beam splitter with several advantages already stated in the Introduction.

In the following section a specific beam-splitter design is considered along with an examination of the effects of limit- ed film-thickness, refractive-index, and incidence-angle errors.

\section{COATED Ge-SLAB BEAM SPLITTER FOR 10.6- $\mu \mathrm{m}$ RADIATION AT $45^{\circ}$ INCIDENCE ANGLE}

As a concrete example, consider the reflection in air $\left(N_{0}=1\right)$ of infrared radiation of wavelength $\lambda=10.6 \mu \mathrm{m}$ (from a $\mathrm{CO}_{2}$ laser) by a planar Ge surface $\left(N_{2}=4\right)$ at an angle of incidence $\phi=45^{\circ}$. To achieve $R_{p}=-R_{s}$ and $T_{p}=T_{s}$, and to realize a beam splitter with excellent characteristics (see Section 1), a single-layer coating is deposited on the Ge substrate with refractive index and thickness given by

$$
N_{1}=1.2824, \quad d=2.4770 \mu \mathrm{m},
$$

as obtained from Eqs. (12) and (2), respectively. The associated polarization-independent reflectance [calculated from Eqs. (19) and (20)] and transmittance ${ }^{8}$ are 17.413 and $82.587 \%$, respectively. By controlling the deposition conditions of $\mathrm{BaF}_{2}$, for example, ${ }^{9}$ the required refractive index of 1.2824 can be attained at $10.6 \mu \mathrm{m}$.

Deviations from the desired conditions of $R_{p}=-R_{s}$ and $T_{p}$ $=T_{s}$ are determined by the behavior of the ratios ${ }^{10}$

$$
\begin{aligned}
& \rho_{r}=R_{p} / R_{s}=\tan \psi_{r} \exp \left(j \Delta_{r}\right), \\
& \rho_{t}=T_{p} / T_{s}=\tan \psi_{t} \exp \left(j \Delta_{t}\right),
\end{aligned}
$$

as a certain design parameter is varied. Ideally, we should have $\psi_{r}=\psi_{t}=45^{\circ}, \Delta_{r}=180^{\circ}$, and $\Delta_{t}=0$.

$R_{p}=-R_{s}$ and $T_{p}=T_{s}$ hold exactly at normal incidence $(\phi$ $\left.=0^{\circ}\right)$ and at the design angle $\left(\phi=45^{\circ}\right)$ and remain approximately correct at all angles in between. This remarkable stationary property of the film-substrate reflection HWR was previously established ${ }^{11}$ and has been verified again for the present example. We find that $\psi_{r}$ and $\psi_{t}$ differ from $45^{\circ}$

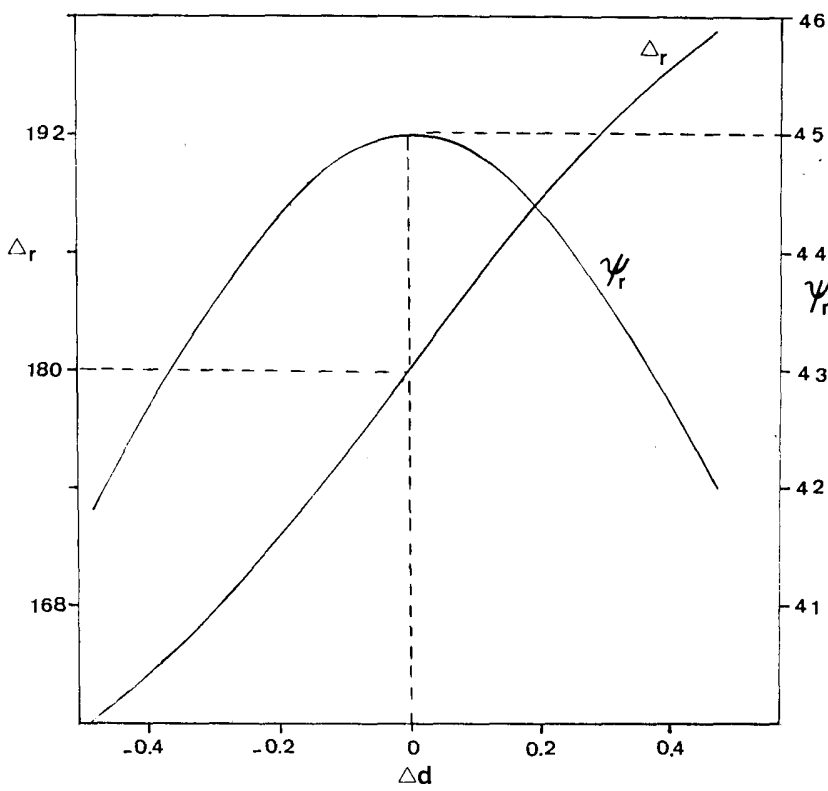

Fig. 3. Reflection ellipsometric angles $\psi_{r}$ and $\Delta_{r}$ (in degrees) plotted versus small changes $\Delta d(\mu \mathrm{m})$ of thickness of a transparent layer [Eqs. (31)] on a Ge substrate that reflects $10.6 \mu \mathrm{m}$ radiation at a $45^{\circ}$ angle of incidence as an HWR and refracts it without change of polarization. $\psi_{r}=45^{\circ}$ and $\Delta_{r}=180^{\circ}$ when $\Delta d=0$, as expected. 


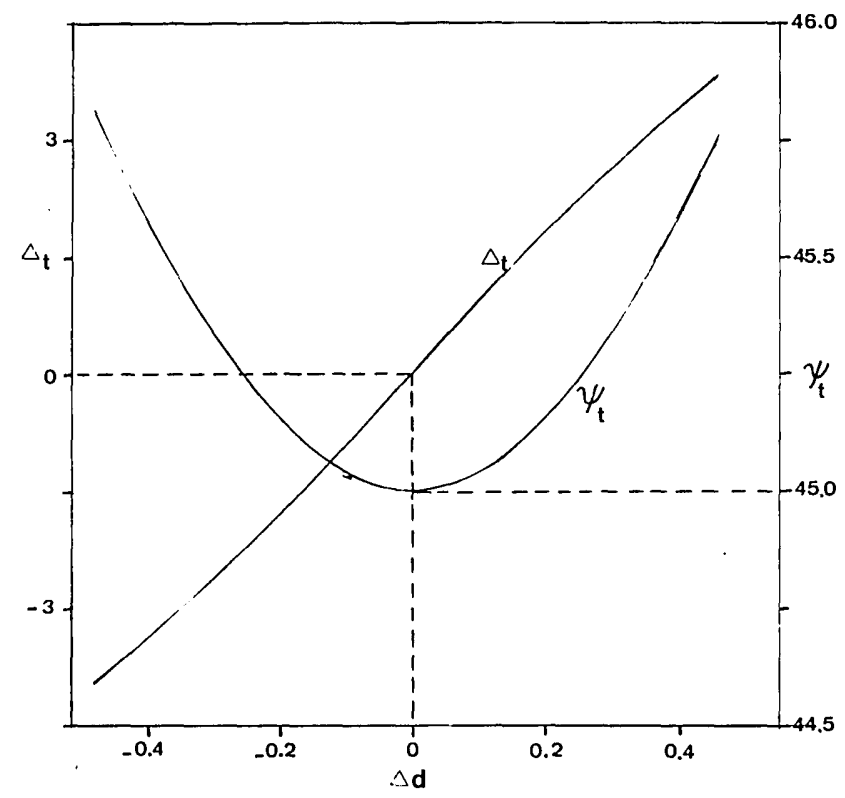

Fig. 4. Same as in Fig. 3 but for the associated transmission ellipsometric angles $\psi_{t}$ and $\Delta_{t}$. In this case, $\psi_{t}=45^{\circ}$ and $\Delta_{t}=0$ when $\Delta d=0$.

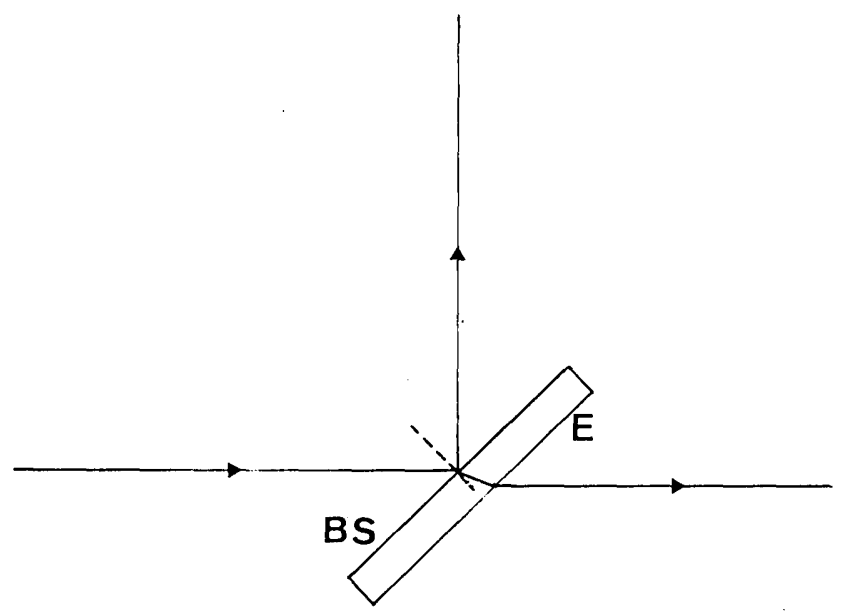

Fig. 5. Dielectric-slab beam splitter at $45^{\circ}$ angle of incidence. The beam-splitting face BS is coated by a single layer to reflect light as a half-wave retarder and refract it without change of polarization. The exit face $\mathrm{E}$ carries a totally refracting, polarization-preserving bilayer.

by $<1^{\circ}, \Delta_{r}-180^{\circ}<5^{\circ}$, and $\Delta_{t}<2^{\circ}$, over the entire range 0 $\leq \phi \leq 45^{\circ}$. All errors change sign as the design angle, $\phi=$ $45^{\circ}$, is passed, and the absolute errors remain within the limits stated above up to $\phi=50^{\circ}$. For $\phi>50^{\circ}$, the errors increase rapidly and become unacceptably large.

Figure 3 shows the effect on $\psi_{r}$ and $\Delta_{r}$ of shifting the film thickness $d$ by as much as $\Delta d= \pm 0.5 \mu \mathrm{m}$ around the design value of $d=2.4770 \mu \mathrm{m}$, and Fig. 4 gives the corresponding results for $\psi_{t}$ and $\Delta_{t}$. Notice that $\psi_{r}$ and $\psi_{t}$ are both locally stationary around $\Delta d=0$, so that the condition $\psi_{r}=\psi_{t}=45^{\circ}$ is insensitive to film-thickness errors, to first order. It is safe to assume that $d$ can be controlled to better than \pm 0.05 $\mu \mathrm{m}$ (or $50 \mathrm{~nm}$ ), which would keep the $\psi$ error below a fraction. of a degree. For the same level of thickness control, $\Delta_{r}$ stays within $\pm 2^{\circ}$ of $180^{\circ}$ and $\Delta_{t}$ within $\pm 0.5^{\circ}$ of 0 . In brief, appreciable film-thickness errors can be tolerated by this design.

Because changes of the wavelength of light around the design wavelength $\lambda=10.6 \mu \mathrm{m}$ have essentially the same effect as equivalent film-thickness errors, neglecting material dispersion, it follows that this beam splitter is reasonably achromatic if the wavelength is scanned over a small range, e.g., between 10 and $11 \mu \mathrm{m}$. This may prove important if the beam splitter is used, e.g., in a Michelson interferometer (Section 6) to examine the spectral composition of the output of a $\mathrm{CO}_{2}$ laser.

At the design point the condition $\psi_{r}=\psi_{t}=45^{\circ}$ is, to first order, unaffected by small shifts of $n_{1}$ around the required value of 1.2824 . However, to limit the associated phase errors to a few degrees requires that $n_{1}$ be controlled to within \pm 0.01 , which is attainable using present-day thinfilm deposition technology.

So far I have mentioned nothing about the exit face of the beam splitter. It is definitely desirable to use a plane-parallel slab (Fig. 5). With the incidence angle being $45^{\circ}$, one beam is reflected at $90^{\circ}$ and the other is transmitted without any angular deviation (but with a small, inconsequential, lateral displacement that is proportional to the thickness of the slab). The beam-splitting face beam splitter is coated by a single. layer with properties given by Eqs. (31) and sensitivity as has already been described. The exit face $\mathbf{E}$ must not produce a spurious change of polarization, or the purpose of the beam splitter will be defeated. This can be accomplished by a transparent $\left(\mathrm{BaF}_{2}-\mathrm{ZnSe}\right)$ bilayer exactly as has been recently described. ${ }^{12}$ Such a bilayer produces total refraction without change of polarization and fits perfectly the purpose of the present beam-splitter design.

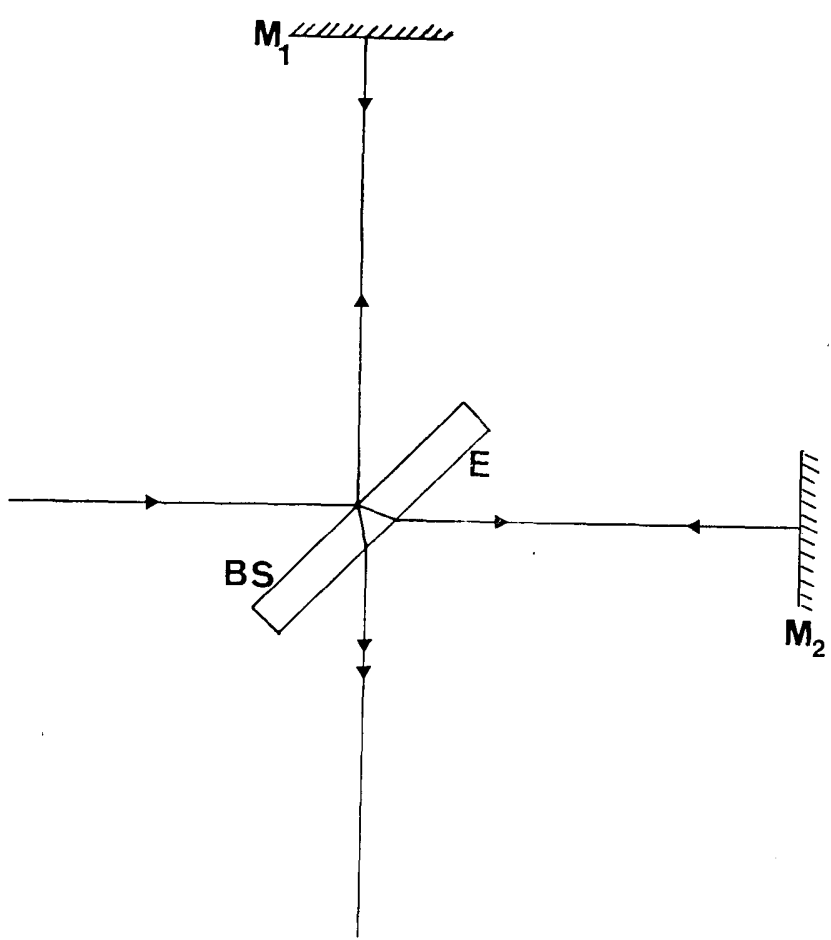

Fig. 6. Polarization-independent Michelson interferometer that uses the beam splitter of Fig. 5. The two recombining beams have equal intensities (when the isotropic return mirrors $M_{1}$ and $M_{2}$ have equal reflectances) and, more importantly, have the same state of polarization as the source beam, irrespective of the latter. 


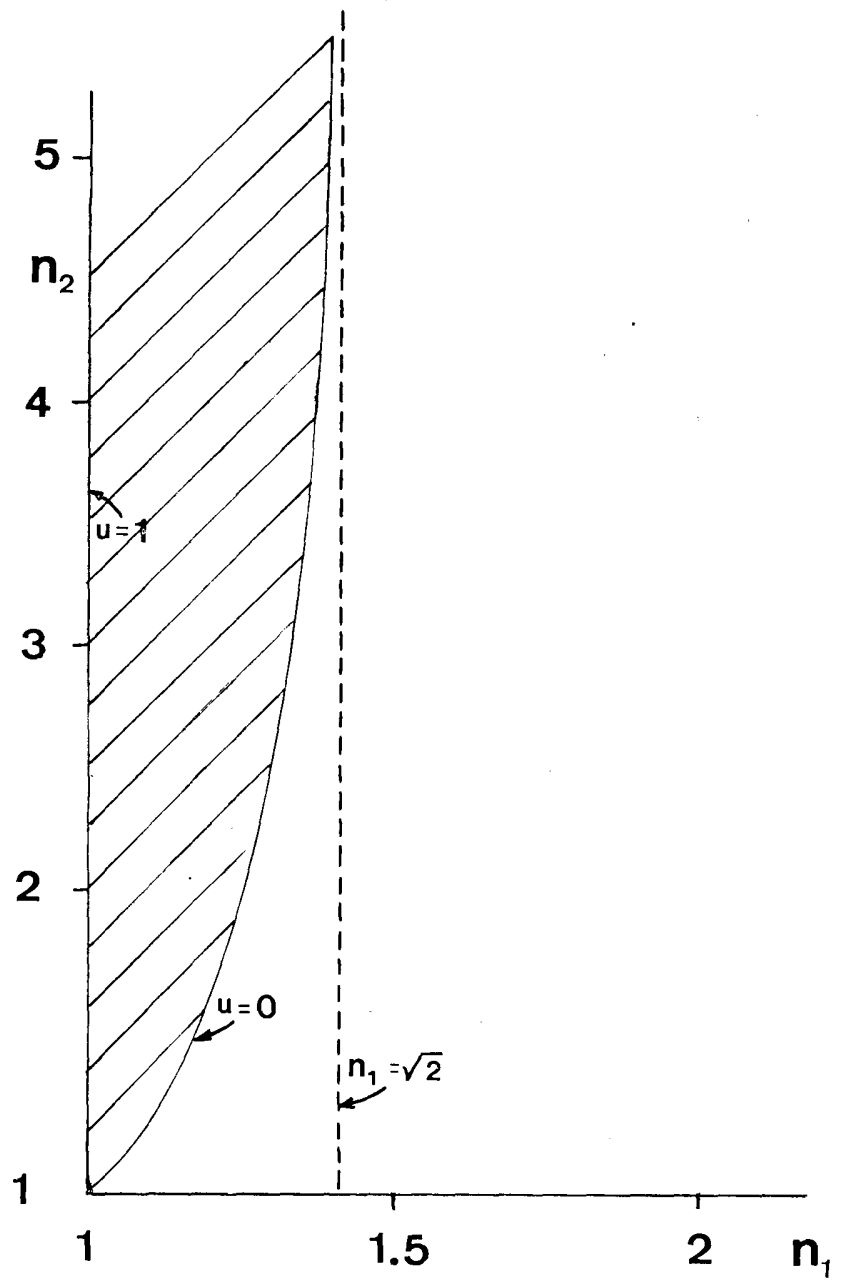

Fig. 7. Domain of all possible pairs of film and substrate refractive indices $\left(n_{1}, n_{2}\right)$ that yield an acceptable answer, $0 \leq u \leq 1$, when substituted into the rhs of Eq. (A2).

\section{MICHELSON INTERFEROMETER}

Figure 6 shows a Michelson interferometer using the new beam splitter. If the effect of every reflection and refraction encountered in the path of each beam between the source and the detector is accounted for, ${ }^{13}$ one can see readily that the two recombining beams (at the detector) are of equal intensity and have the same polarization as that of the incident beam, independent of the input polarization. We are unaware of any other equally simple beam-splitter design that imparts this remarkable property to the Michelson interferometer. ${ }^{14}$

\section{APPENDIX A}

For a given, all-dielectric, film-substrate system with known refractive indices $n_{1}$ and $n_{2}$ it is possible to determine the angle of incidence, $\phi=\phi_{\mathrm{HWR}}$, at which half-wave retardation is attained in reflection with a layer of quarter-wave optical thickness at that angle. Equation (12) permits an explicit solution for $\phi$. If we define

$$
u=\sin ^{2} \phi,
$$

then Eq. (12) can be manipulated to yield the answer

$$
u=\left(n_{1}^{4} n_{2}{ }^{2}+n_{1}^{4}-2 n_{1}{ }^{2} n_{2}{ }^{2}\right) /\left(n_{1}^{4}-n_{2}{ }^{2}\right) .
$$

The refractive-index pair $\left(n_{1}, n_{2}\right)$ must be such that Eq. (A2) gives

$$
0 \leq u \leq 1 \text {. }
$$

The limiting cases of $u=0$ and $u=1$ of Eq. (A2) define the two boundaries

$$
\begin{aligned}
& n_{1}=\sqrt{2} n_{2} /\left(n_{2}^{2}+1\right)^{1 / 2}, \\
& n_{1}=1
\end{aligned}
$$

of the domain of the $n_{1} n_{2}$ plane of all possible pairs $\left(n_{1}, n_{2}\right)$ that lead to HWR in reflection and the effect described in this paper. The boundary curve of Eq. (A4) has the line $n_{1}=$ $\sqrt{2}$ as an asymptote (as $n_{2} \rightarrow \infty$ ), so that the solution domain lies entirely in the strip $1<n_{1}<\sqrt{2}$ of the $n_{1} n_{2}$ plane, as is shown in Fig. 7.

For a transparent film on an absorbing substrate with complex refractive index $n_{2}-j k_{2}$, the analytical solution of this appendix gives an accurate estimate of $\phi_{\mathrm{HWR}}$ when $k_{2} \ll$ $n_{2}$. The latter condition holds for semiconductors over an appreciable part of the visible spectrum. ${ }^{15}$ If $k_{2}$ cannot be neglected (e.g., for metallic substrates), then the generally applicable numerical approach of Ref. 11 ought to be used.

\section{ACKNOWLEDGMENTS}

The kind hospitality of the Département de Physique du Solide, Université de Provence (where this work was completed), a sabbatical from the University of New Orleans, and a Fulbright Senior Research Scholar Award are all gratefully acknowledged.

\section{REFERENCES AND NOTES}

1. R. M. A. Azzam, "Simultaneous reflection and refraction of light without change of polarization by a single-layer-coated dielectric surface," Opt. Lett. 10, 107-109 (1985).

2. R. M. A. Azzam, "Variable-reflectance thin-film polarizationindependent beam splitters for 0.6328 - and $10.6-\mu \mathrm{m}$ laser light," Opt. Lett. 10, 110-112 (1985).

3. This is exactly the same polarization transformation that occurs when light is reflected at normal incidence from an optically isotropic surface. See R. M. A. Azzam, "The change of polarization that accompanies normal-incidence reflection from a passive isotropic surface," Optik 63, 37-41 (1982).

4. R. H. Muller, "Definitions and conventions in ellipsometry," Surf. Sci. 16, 14-33 (1969).

5. See, e.g., H. K. Pulker, "Characterization of optical thin films," Appl. Opt. 18, 1969-1977 (1979).

6. Except, of course, if a dense medium of incidence, e.g., glass, is used instead of air.

7. See. Eqs. (9) and (10) of Ref. 1.

8. The polarization-independent intensity transmittance for this lossless film-substrate system is given by $\tau=1-\mathcal{R}$, where $\mathcal{R}$ is the reflectance plotted in Fig. 2.

9. P. C. Kemeny, "Refractive index of thin films of barium fluoride," Appl. Opt. 21, 2052-2056 (1982).

10. The ellipsometric angles $\left(\psi_{r}, \Delta_{r}\right)$ and $\left(\psi_{t}, \Delta_{t}\right)$ determine the change of polarization on reflection and transmission, respectively.

11. R. M. A. Azzam and M. E. R. Khan, "Single-reflection filmsubstrate half-wave retarders with nearly stationary properties over a wide range of incidence angles," J. Opt. Soc. Am. 73, 160166 (1983). Dielectric, semiconducting, and metallic substrates were considered in this paper.

12. R. M. A. Azzam and K. Javily, "Total refraction at oblique 
incidence by a transparent bilayer coating on a high-index transparent or absorbing substrate," Appl. Opt. 24, 4454-4459 (1985). The outer film of the bilayer on the exit face should have the lower refractive index of 1.2070 , requiring a change of the evaporation conditions of $\mathrm{BaF}_{2}$ (Ref. 9).

13. We assume that mirrors $M_{1}$ and $M_{2}$ are optically isotropic and have equal reflectances, say, $\mathcal{R}_{\mathrm{M}}$. Normal-incidence reflection from such mirrors is accompanied by the HWR, Ref. 3 . Both external and internal reflections at the beam-splitting face also cause HWR. Total refraction at the exit face (caused by the bilayer coating) is, of course, inconsequential. Each of the recombining beams suffers two HWR reflections leading to a net null effect on polarization, and each suffers the same total intensity loss, given by the factor $\mathcal{R}_{\mathrm{BS}}\left(1-\mathcal{R}_{\mathrm{BS}}\right) \mathcal{R}_{\mathrm{M}}$, where $\mathcal{R}_{\mathrm{BS}}$ and $\left(1-\mathscr{R}_{\mathrm{BS}}\right)$ are the reflectance and transmittance of the beam-splitting face, respectively.

14. Two publications have been brought to our attention recently that discuss polarization-independent beam splitters. They are A. A. M. Saleh, "Polarization-independent, multilayer dielectrics at oblique incidence," Bell Syst. Tech. J. 54, 1027-1049 (1975); E. E. Bergmann, "Polarization-independent beam splitter," Rev. Sci. Instrum. 54, 579-581 (1983). These complement the references already cited in Refs. 1 and 2.

15. D. E. Aspnes and A. A. Studna, "Dielectric functions and optical parameters of $\mathrm{Si}, \mathrm{Ge}, \mathrm{GaP}, \mathrm{GaAs}, \mathrm{GaSb}, \mathrm{InP}$, InAs, and InSb from 1.5 to $6.0 \mathrm{eV}$," Phys. Rev. B 27, 985-1009 (1983). 